\title{
Clinical significance of the gene expression profile in residual tumor cells after neoadjuvant chemo- radiotherapy for esophageal cancer
}

\author{
KOJI TANAKA ${ }^{1}$, KOHHEI OTAKE ${ }^{1}$, YASUHIKO MOHRI ${ }^{1}$, MASAKI OHI ${ }^{1}$, TAKESHI YOKOE ${ }^{1}$, \\ YUJI TOIYAMA ${ }^{1}$, CHIKAO MIKI $^{1}$, HITOSHI TONOUCHI ${ }^{2}$ and MASATO KUSUNOKI ${ }^{1,2}$
}

Departments of ${ }^{1}$ Gastrointestinal and Pediatric Surgery and ${ }^{2}$ Innovative Surgery, Division of Reparative Medicine, Institute of Life Sciences, Mie University Graduate School of Medicine, 2-174 Edobashi, Tsu, Mie 514-8507, Japan

Received January 2, 2009; Accepted February 27, 2009

DOI: 10.3892/or_00000379

\begin{abstract}
Recurrence after neoadjuvant chemoradiotherapy (CRT) followed by surgery is high in patients with esophageal cancer. No standard second line therapy is currently available for patients with recurrence. This study aimed to evaluate the expression of chemo-radiosensitive genes after neoadjuvant CRT in residual tumor cells. Thirteen patients with esophageal squamous cell carcinoma underwent 5-fluorouracil (5-FU) and cisplatin (CDDP) based CRT followed by surgery. Total RNA was successfully obtained from 6 formalin-fixed paraffin-embedded (FFPE) specimens using proteinase $\mathrm{K}$ digestion and phenol chloroform extraction. TS and DPD as the 5-FU pathway gene, ERCC1 as the CDDP pathway gene, and EGFR, VEGF, HIF1a as radioresistant genes were measured using real-time reverse transcription polymerase chain reaction; comparing the mRNA level of each gene in pre-CRT biopsy with that in post-CRT FFPE specimens. Five patients had less than one-third residual tumor cells in resected specimens histopathologically; eight had more than twothirds residual tumor cells. There were significant increases in TS $(\mathrm{p}=0.02)$ and DPD $(\mathrm{p}=0.01)$ levels in residual tumor cells after CRT. Significant decreases in ERCC1 $(p=0.03)$, EGFR $(p=0.01)$, VEGF $(p=0.003)$ and HIF1a $(p=0.003)$ levels were observed. 5-FU and CDDP based CRT up-regulated 5-FU pathway genes and down-regulated CDDP pathway and radioresistant genes. The expression of chemo-radiosensitive genes was significantly changed in residual tumor cells after CRT. Gene expression analysis of residual tumor cells in FFPE specimens may be useful when selecting a second line
\end{abstract}

Correspondence to: Dr Koji Tanaka, Department of Gastrointestinal and Pediatric Surgery, Division of Reparative Medicine, Institute of Life Sciences, Mie University Graduate School of Medicine, 2-174 Edobashi, Tsu, Mie 514-8507, Japan

E-mail: qouji@clin.medic.mie-u.ac.jp

Key words: microdissection, chemoradiotherapy, esophageal cancer chemotherapy regimen for recurrent esophageal cancer after CRT.

\section{Introduction}

Neoadjuvant chemoradiotherapy (CRT) followed by surgery has been shown to reduce incomplete local resection, local and systemic recurrences, and to improve disease-free and overall survival in patients with esophageal cancer (1-3).

However, patients with an incomplete pathological response still have a poorer prognosis because of higher locoregional recurrence and distant metastasis compared to those with a complete pathological response (pCR) (4-6). Since the pCR rate is only approximately $30 \%$ (6), most patients $(70 \%)$ receiving neoadjuvant CRT followed by surgery have a high risk of disease recurrence and thus will need adjuvant therapy such as additional radiotherapy or chemotherapy.

Recently, histopathologic response based on the quantification of residual tumor cells has been shown to be related to recurrence or survival in esophageal cancer patients receiving CRT followed by surgery (7-9). However, it is difficult to determine the difference between CRT-induced fibrosis and pre-existing tumor desmoplasia when quantifying the proportion of residual tumor cells (9).

In this study, instead of quantifying the proportion of residual tumor cells, we focused on the gene expression of residual tumor cells in formalin-fixed paraffin-embedded (FFPE) specimens after neoadjuvant CRT. Microdissection and real-time PCR were used for gene expression analysis of residual tumor cells in FFPE specimens, because residual tumor cells in each specimen are small and real-time PCR is relatively uniform compared with immunohistochemistry (10-13).

This preliminary study aimed to evaluate the expressions of chemo-radiosensitive genes in residual tumor cells of esophageal cancer after neoadjuvant CRT. We evaluated thymidylate synthase (TS) and dihydropyrimidine dehydrogenase (DPD) as 5-FU pathway genes, excision repair crosscomplementation group 1 (ERCC1) as a CDDP pathway gene, and epidermal growth factor receptor (EGFR), vascular 
Table I. Primer and probe sequences of target genes.

\begin{tabular}{|c|c|c|}
\hline Gene & Primer and probe & Sequence \\
\hline \multirow[t]{3}{*}{ TS } & Forward primer & 5'-GCCTCGGTGTGCCTTTCA-3' \\
\hline & Reverse primer & 5'-CCCGTGATGTGCGCAAT-3' \\
\hline & Probe & 5'-TCGCCAGCTACGCCCTGCTCA-3' \\
\hline \multirow[t]{3}{*}{ DPD } & Forward primer & 5'-AGGACGCAAGGAGGGTTTG-3' \\
\hline & Reverse primer & 5'-GTCCGCCGAGTCCTTACTGA-3' \\
\hline & Probe & 5'-CAGTGCCTACAGTCTCGAGTCTGCCAGTG-3' \\
\hline \multirow[t]{3}{*}{ ERCC1 } & Forward primer & 5'-GGGAATTTGGCGACGTAATTC-3' \\
\hline & Reverse primer & 5'-GCGGAGGCTGAGGAACAG-3' \\
\hline & Probe & 5'-CACAGGTGCTCTGGCCCAGCACATA-3' \\
\hline \multirow[t]{3}{*}{ VEGF } & Forward primer & 5'-CAGAAGGAGGAGGGCAGAA-3' \\
\hline & Reverse primer & 5'-CTCGATTGGATGGCAGTAGC-3' \\
\hline & Probe & 5'-TCCATGAACTTCACCACTTCGTGATGA-3' \\
\hline \multirow[t]{3}{*}{ HIF1a } & Forward primer & 5'-CCGCTGGAGACACAATCATA-3' \\
\hline & Reverse primer & 5'-CTTCCTCAAGTTGCTGGTCA-3' \\
\hline & Probe & 5'-TGGCAGCAACGACACAGAAACTGA-3' \\
\hline \multirow[t]{3}{*}{ EGFR } & Forward primer & 5'-ССТАTGTGCAGAGGAATTATGATCTTT-3' \\
\hline & Reverse primer & 5'-CCACTGTGTTGAGGGCAATG-3' \\
\hline & Probe & 5'-AACCAGCCACCTCCTGGATGGTCTTTAA-3' \\
\hline \multirow[t]{3}{*}{ B-actin } & Forward primer & 5'-ACAGAGCCTCGCCTTTGC-3' \\
\hline & Reverse primer & 5'-GCGGCGATATCATCATCC-3' \\
\hline & Probe & 5'-CCGCCGCCAGCTCACCAT-3' \\
\hline
\end{tabular}

endothelial growth factor (VEGF), and hypoxia-inducible factor- $1 \alpha(\mathrm{HIF} 1 \mathrm{a})$ as radioresistant genes.

\section{Materials and methods}

Patients and specimens. In this preliminary analysis, 13 patients with histologically proven esophageal squamous cell carcinoma who received neoadjuvant chemo-radiotherapy (CRT) followed by surgery were selected because of the availability of pre-CRT endoscopic biopsy specimens. Before pre-CRT endoscopic biopsy, all patients signed informed consent forms for their tissue to be used in this study. PreCRT biopsy specimens were snap-frozen in liquid nitrogen and kept at $-80^{\circ} \mathrm{C}$ until use. All resected specimens were fixed in $10 \%$ formalin, and embedded in paraffin.

5-Fluorouracil and cisplatin-based chemo-radiotherapy regimen. All patients received systemic 5-FU and CDDP chemotherapy with concurrent radiotherapy. The regimen included four cycles of 5-FU given as $600 \mathrm{mg} / \mathrm{m}^{2}$ administered intravenously for $24 \mathrm{~h}$, and UFT (Tegafur and Uracil) given as $400 \mathrm{mg} /$ body weight administered orally for 5 days, plus CDDP given as 2-4 mg/day administered intravenously for 5 days with concurrent 40 Gy radiation followed by esophageal resection. Preoperative radiotherapy was delivered to both primary tumor and peritumoral area at a dose of $40 \mathrm{~Gy}$ in 20 fractions within 4 weeks ( 2 Gy/day for 5 days a week). The time interval between neoadjuvant CRT and surgery was 2-3 weeks.

Clinical and histopathologic response. Tumors were staged based on the Guidelines for the Clinical and Pathologic Studies on Carcinoma of the Esophagus (14). Clinical response was evaluated by barium swallow, endoscopy and computed tomography and graded as complete response (CR), partial response (PR), no change (NC) or progressive disease (PD) (14). The degree of histopathologic response was classified into four categories: grade 0 , neither necrosis nor regressive changes; grade $1,>2 / 3$ vital residual tumor cells (VRTCs); grade 2, <1/3 VRTCs; or grade 3, no VRTCs (14).

RNA extraction from pretreatment biopsy specimens. PreCRT endoscopic biopsy specimens were homogenized with a Mixer Mill MM 300 homogenizer (Qiagen, Inc., Chatsworth, CA). Total RNA was isolated using an RNeasy mini kit (Qiagen, Inc.) according to the manufacturer's instructions.

Microdissection in formalin-fixed, paraffin-embedded (FFPE) specimens. Sections (10- $\mu \mathrm{m}$ thick) of formalin-fixed, paraffin-embedded (FFPE) specimens were stained with nuclear fast red and subsequently manually microdissected to collect residual tumor cells, with reference to hematoxylin and eosin sections. 
Table II. Clinical and histopathologic responses.

\begin{tabular}{ll}
\hline A. Clinical response & No. of patients \\
\hline Complete response & 1 \\
Partial response & 9 \\
No change & 3 \\
Progressive disease & 0 \\
\hline
\end{tabular}

B. Histopathologic response

No. of patients

\begin{tabular}{ll}
\hline Grade 3 & 0 \\
Grade 2 , residual tumor $<\mathrm{l} / 3$ & 5 \\
Grade $\mathrm{lb}, 1 / 3<$ residual tumor $<2 / 3$ & 5 \\
Grade $1 \mathrm{a}, 2 / 3<$ residual tumor & 3 \\
Grade 0 & 0 \\
\hline
\end{tabular}

RNA extraction from FFPE specimens. Microdissected samples were digested with proteinase $\mathrm{K}$ in lysis buffer containing Tris-HCl, EDTA, and sodium dodecyl sulfate as previously reported with minor modification (15). RNA was purified by phenol and chloroform extraction.

cDNA synthesis. cDNA was synthesized with random hexamer primer and Superscript III reverse transcriptase (Invitrogen, Carlsbad, CA) according to the manufacturer's instructions.

Real-time quantitative RT-PCR. Real-time quantitative RTPCR analysis was done using an ABI PRISM 7700 Sequence Detection System (Applied Biosystems, Inc., Foster City, CA). Primers and probes for B-actin, VEGF and HIF1a were designed with primer 3 software (Biology Workbench Version 3.2, San Diego Supercomputer Center, at the University of California, San Diego). Primers and probes for TS, DPD, ERCC1 and EGFR were synthesized according to previously published sequences (16). Sequences are shown in Table I. PCR was done in a final volume of $25 \mu 1$ with a Taqman Universal PCR Master Mix (Applied Biosystems) using $0.5 \mu 1$ cDNA, $900 \mathrm{nM}$ of each primer, and $200 \mathrm{nM}$ of probe for the respective genes. Cycling conditions were $50^{\circ} \mathrm{C}$ for $2 \mathrm{~min}$ and $95^{\circ} \mathrm{C}$ for $10 \mathrm{~min}$ followed by 40 cycles at $95^{\circ} \mathrm{C}$ for $15 \mathrm{sec}$ and $60^{\circ} \mathrm{C}$ for $1 \mathrm{~min}$.

Relative expression levels of TS, DPD, ERCC1, EGFR, $V E G F$ and HIF la. Relative gene expression levels were determined by the standard curve method. The standard curves and line equations were generated using 5-fold serially diluted solutions of cDNA from the esophageal cancer cell line TE1. All standard curves were linear in the analyzed range with an acceptable correlation coefficient $\left(\mathrm{R}^{2}\right)$. The amount of target gene expression was calculated from the standard curve. Quantitative normalization of cDNA in each sample was performed using the expression of the $\beta$-actin gene as an internal control. Finally, target gene mRNA levels were given as ratios to $\beta$-actin mRNA levels. Real-time PCR assays were done in duplicate for each sample and mean values used for calculations of the mRNA expression levels.

Statistical analysis. All statistical analyses were done using JMP version 5 (SAS Institute, Inc. Cary, NC, USA). Results were expressed as median value (inter-quartile range). The Wilcoxon rank sum test was used to compare between preand post-CRT tumoral mRNA levels.

Box and whisker plots were used to summarize the distribution of mRNA level in cancer tissue of pre- and postCRT. The horizontal line in the box represents the 50th (median) and the upper and lower lines of the box represent 75th and 25th quartiles, respectively. The whiskers indicate the range of the measurements. P-values $<0.05$ were considered statistically significant.

\section{Results}

Patient characteristics and CRT response. The 13 ESCC patients were 11 males and 2 females, median age 66 years (range 53-77). Clinical and histopathologic responses of the 13 patients are shown in Table IIA and IIB, respectively. One patient was classified as complete response, 9 as partial response, and 3 as no change. According to the histopathological response criteria, 5 of the 13 patients who underwent CRT followed by esophageal resection were categorized as grade 2 and 8 as grade 1 . Seven $(54 \%)$ of the 13 patients were pathologically down-staged.

Table III. Clinical outcome of 6 patients after neoadjuvant chemoradiotherapy followed by surgery.

\begin{tabular}{cccccrc}
\hline $\begin{array}{l}\text { No. of } \\
\text { patients }\end{array}$ & Age & Gender & $\begin{array}{c}\text { Clinical } \\
\text { stage }\end{array}$ & $\begin{array}{c}\text { Pathological } \\
\text { stage }\end{array}$ & $\begin{array}{c}\text { Clinical } \\
\text { response }\end{array}$ & $\begin{array}{c}\text { Histopathologic } \\
\text { response }\end{array}$ \\
\hline 3 & 71 & M & T3NOMO Stage 2 & T3NOMO Stage 2 & NC & Grade 1 \\
4 & 53 & M & T4N2MO Stage 3 & T4N2MO Stage 3 & PR & Grade 1 \\
5 & 67 & M & T4N2MO Stage 4a & T2N2MO Stage 3 & PR & Grade 1 \\
6 & 69 & M & T3N4MO Stage 4a & T3N4MO Stage 4a & PR & Grade 1 \\
7 & 63 & M & T3N2MO Stage 3 & T2NOMO Stage 2 & PR & Grade 1 \\
10 & 68 & F & T3N2MO Stage 3 & T2NOMO Stage 2 & PR
\end{tabular}


TS

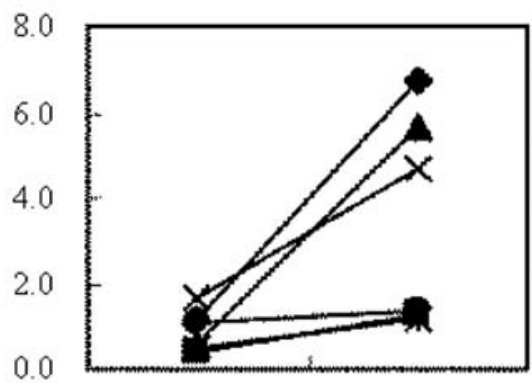

Pre-CRT Post-CRT

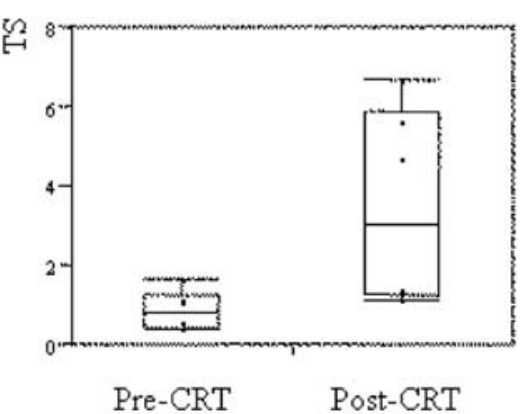

$\mathrm{p}=0.02$

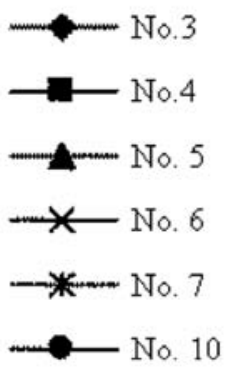

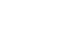
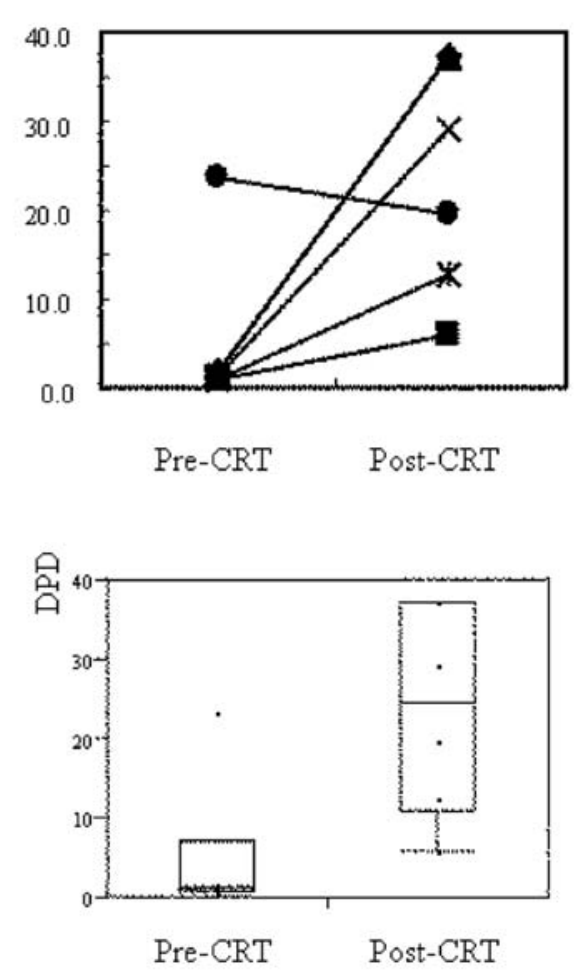

$\mathrm{p}=0.01$

Figure 1. Changes in TS and DPD as a 5-FU pathway gene during CRT.

\section{ERCC1}
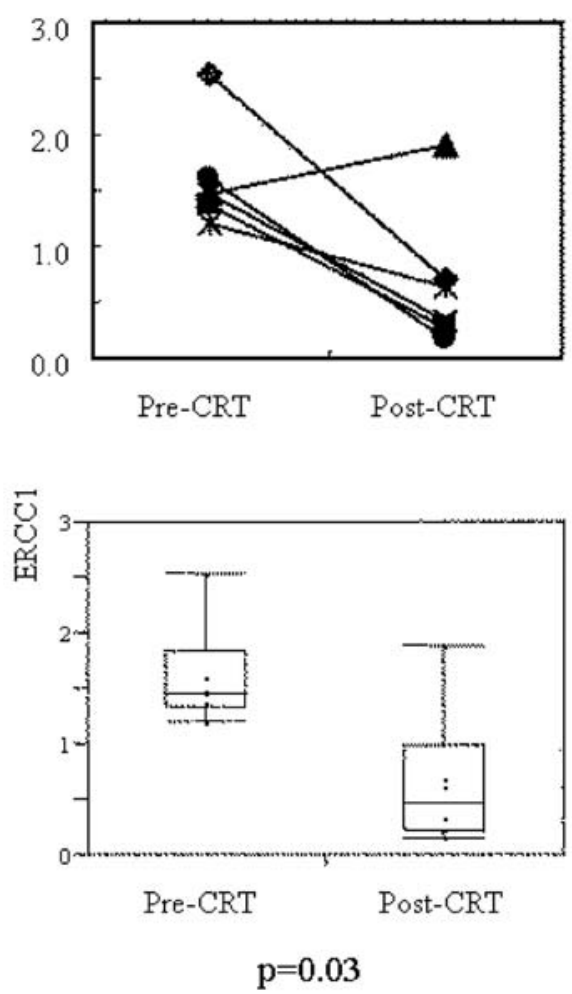

Figure 2. Changes in ERCC1 as a CDDP pathway gene during CRT.
Microdissection and RNA extraction from FFPE specimens. Five patients had less than one-third residual tumor cells (grade 2) in their resected specimens, histopathologically and 8 had $>2 / 3$ residual tumor cells (grade 1 ).

Total RNA extraction was successful in 6 of the 13 FFPE specimens (Table III). It was difficult for us to microdissect residual tumor cells and to extract RNA from FFPE specimens in patients with grade 2 histopathologic responses. Thereafter, we compared the mRNA level of each gene in pre-CRT biopsy with that in post-CRT FFPE specimens in these 6 patients.

5-FU pathway gene level during CRT. TS and DPD were measured as the 5-FU pathway gene. TS levels were 0.82 (0.44-1.26) in pre-CRT and 3.06 (1.27-5.89) in post-CRT, respectively. DPD levels were $1.52(1.06-7.13)$ in pre-CRT and 24.51 (10.94-37.27) in post-CRT, respectively. There were significant increases in TS $(\mathrm{p}=0.02)$ and DPD $(\mathrm{p}=0.01)$ mRNA levels in residual tumor cells after CRT (Fig. 1). One of the 6 patients had decreased DPD expression in residual tumor cells.

CDDP pathway gene level during CRT. ERCC1 was measured as the CDDP pathway gene. ERCC1 levels were 1.47 (1.34-1.84) in pre-CRT and 0.48 (0.23-0.99) in postCRT, respectively. A significant decrease in ERCC1 mRNA level was observed ( $p=0.03$ ) (Fig. 2). One of the 6 patients had increased ERCC1 expression in residual tumor cells. 
VEGF

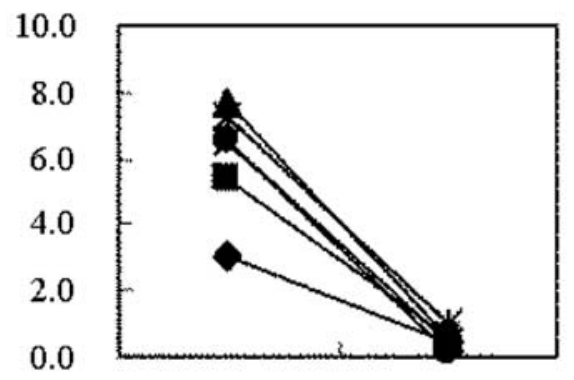

Pre-CRT Post-CRT

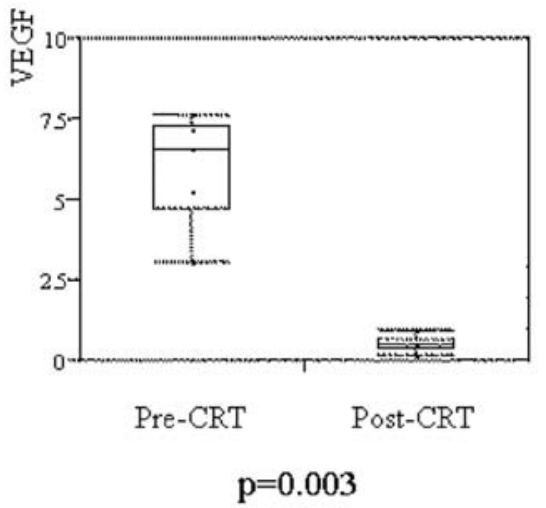

HIFla
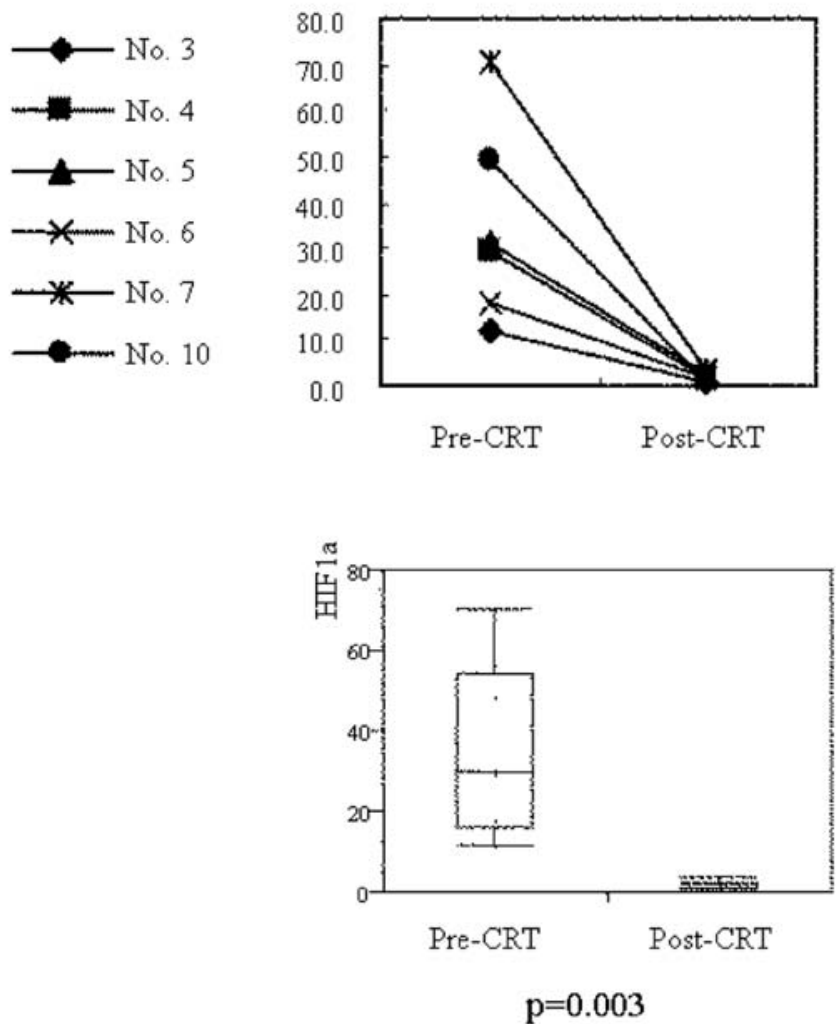

Figure 3. Changes in VEGF and HIF1a as radioresistance genes during CRT

\section{EGFR}

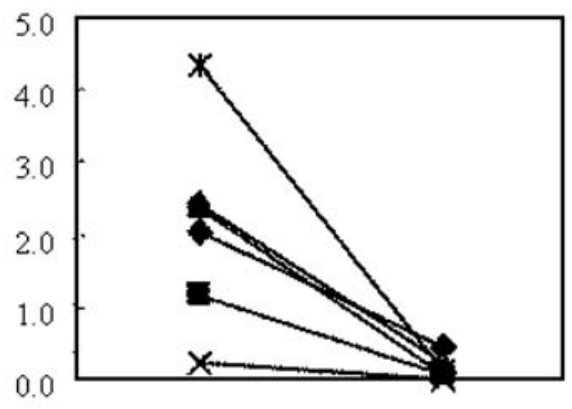

Pre-CRT

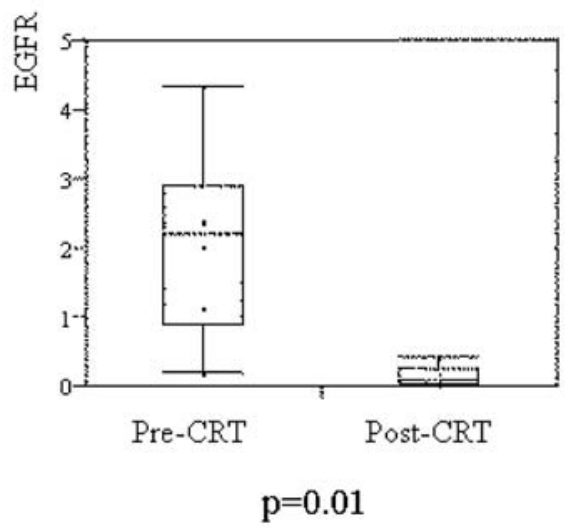

Figure 4. Changes in EGFR as a radioresistance gene during CRT.
Radioresistance gene level during CRT. EGFR levels were $2.21(0.91-2.90)$ in pre-CRT and $0.09(0.03-0.26)$ in post-CRT, respectively. VEGF levels were 6.57 (4.75-7.30) in pre-CRT and $0.53(0.40-0.68)$ in post-CRT, respectively. HIF1a levels were 30.01 (16.25-54.24) in pre-CRT and 1.84 (1.03-2.62) in post-CRT, respectively. VEGF and HIF1a were measured as radioresistant genes. There were significant decreases in VEGF ( $\mathrm{p}=0.003)$ and HIF1a $(\mathrm{p}=0.003)$ mRNA levels in residual tumor cells after CRT (Fig. 3). EGFR was also measured as a radioresistant gene. A significant decrease in EGFR mRNA level was observed ( $\mathrm{p}=0.01$ ) (Fig. 4).

\section{Discussion}

This preliminary study showed that expressions of chemoradiosensitive genes were changed by CRT in residual tumor cells of esophageal cancer. When comparing mRNA levels in pre-CRT biopsy and in post-CRT FFPE specimens, TS and DPD were significantly up-regulated in residual tumor cells, while ERCC1, VEGF, HIF1a and EGFR were significantly down-regulated.

As noted by Liersch et al (13), changes in TS expression during CRT may be explained by: i) CRT-induced upregulation of TS, ii) a selection process wherein tumor cells with low TS responded to 5-FU and were then eradicated or iii) a combination of i) and ii). DPD may be explained in the same manner as TS. Tumor cells with high TS and DPD seem to be resistant to 5-FU (17), and thus may be regarded as residual tumor cells. 
Tumors with low ERCC1 have been reported to be associated with favorable responses to CDDP (18). Overexpression of EGFR, VEGF or HIF1a seems correlated with chemotherapy or radiation resistance in several malignancies (19-21). EGFR inhibitors have been shown to enhance the effects of chemotherapy or radiation (18). HIF inhibitor has also been anticipated to be a novel molecular targeted agent, especially for HIF 1 overexpressing tumor cells (21).

Recent studies have focused on inhibiting chemoradioresistance genes by using antisense oligonucleotides or small interfering RNA designed to inhibit target genes to sensitize cancer cells to radiation or chemotherapeutic drugs. Our results showed that CRT itself decreased the expression of chemo-radioresistance genes. Therefore, it is possible that CDDP and radiation may still be appropriate treatments, or can be used for recurrent esophageal cancer after CRT. Since a number of genes, except for these genes, are related to 5-FU, CDDP, and radiation sensitivity (22), this speculation is over simplistic. However, we believe that analysis of gene expression profiles using post-CRT samples would be useful for the treatment of recurrence after neoadjuvant CRT and surgery.

In vitro study using ESCC lines showed that CRT also up-regulated or down-regulated the expression of chemoradiosensitive genes, as in clinical specimens (data not shown). However, there was a discrepancy between the results of in vitro study and those of clinical specimens; thus, it may depend on the type of cell line or difference in CRT setting.

As described in previous reports (4-6), the pCR rate is now just $30 \%$ and approximately $70 \%$ of patients receiving neoadjuvant CRT followed by surgery have a high risk of locoregional and distant metastatic recurrence with poorer prognosis. To improve overall survival of esophageal cancer patients, we need to also focus on treatment strategies for patients with incomplete pathological responses.

In conclusion, expressions of TS, DPD, ERCC1, EGFR, VEGF and HIF1a were significantly changed by neoadjuvant 5-FU and CDDP based CRT in residual tumor cells. As well as quantification of the histopathologic response, gene expression analysis in residual tumor cells may provide significant information on a suitable strategy for patients with recurrent esophageal cancer after neoadjuvant CRT followed by surgery.

\section{References}

1. Urschel JD and Vasan H: A meta-analysis of randomized controlled trials that compared neoadjuvant chemoradiation and surgery to surgery alone for resectable esophageal cancer. Am J Surg 185: 538-543, 2003.

2. Berger AC, Farma J, Scott WJ, et al: Complete response to neoadjuvant chemoradiotherapy in esophageal carcinoma is associated with significantly improved survival. J Clin Oncol 23: 4330-4337, 2005.

3. Mariette C, Piessen G, Lamblin A, Mirabel X, Adenis A and Triboulet JP: Impact of preoperative radiochemotherapy on postoperative course and survival in patients with locally advanced squamous cell oesophageal carcinoma. Br J Surg 93: 1077-1083, 2006.

4. Rohatgi PR, Swisher SG, Correa AM, et al: Failure patterns correlate with the proportion of residual carcinoma after preoperative chemoradiotherapy for carcinoma of the esophagus. Cancer 104: 1349-1355, 2005.
5. Hermann RM, Horstmann O, Haller F, et al: Histomorphological tumor regression grading of esophageal carcinoma after neoadjuvant radiochemotherapy: which score to use? Dis Esophagus 19: 329-334, 2006.

6. Hammoud ZT, Kesler KA, Ferguson MK, et al: Survival outcomes of resected patients who demonstrate a pathologic complete response after neoadjuvant chemoradiation therapy for locally advanced esophageal cancer. Dis Esophagus 19: 69-72, 2006.

7. Chirieac LR, Swisher SG, Ajani JA, et al: Posttherapy pathologic stage predicts survival in patients with esophageal carcinoma receiving preoperative chemoradiation. Cancer 103: 1347-1355, 2005.

8. Schneider PM, Baldus SE, Metzger R, et al: Histomorphologic tumor regression and lymph node metastases determine prognosis following neoadjuvant radiochemotherapy for esophageal cancer: implications for response classification. Ann Surg 242: 684-692, 2005.

9. Brucher BL, Becker K, Lordick F, et al: The clinical impact of histopathologic response assessment by residual tumor cell quantification in esophageal squamous cell carcinomas. Cancer 106: 2119-2127, 2006.

10. Jakob C, Aust DE, Meyer W, et al: Thymidylate synthase, thymidine phosphorylase, dihydropyrimidine dehydrogenase expression, and histological tumour regression after 5-FU-based neo-adjuvant chemoradiotherapy in rectal cancer. J Pathol 204: 562-568, 2004.

11. Jakob C, Liersch T, Meyer W, et al: Immunohistochemical analysis of thymidylate synthase, thymidine phosphorylase, and dihydropyrimidine dehydrogenase in rectal cancer (cUICC II/ III): correlation with histopathologic tumor regression after 5fluorouracil-based long-term neoadjuvant chemoradiotherapy. Am J Surg Pathol 29: 1304-1309, 2005.

12. Jakob C, Liersch T, Meyer W, et al: Prognostic value of histologic tumor regression, thymidylate synthase, thymidine phosphorylase, and dihydropyrimidine dehydrogenase in rectal cancer UICC Stage II/III after neoadjuvant chemoradiotherapy. Am J Surg Pathol 30: 1169-1174, 2006.

13. Liersch T, Langer C, Ghadimi BM, et al: Lymph node status and TS gene expression are prognostic markers in stage II/III rectal cancer after neoadjuvant fluorouracil-based chemoradiotherapy. J Clin Oncol 24: 4062-4068, 2006.

14. Japanese Society for Esophageal Disease: Guidelines for the clinical and pathologic studies on carcinoma of the esophagus, 9th edition. Kanehara: Japanese Society for Esophageal Disease, 1999.

15. Bijwaard KE, Aguilera NS, Monczak Y, Trudel M, Taubenberger JK and Lichy JH: Quantitative real-time reverse transcription-PCR assay for cyclin D1 expression: utility in the diagnosis of mantle cell lymphoma. Clin Chem 47: 195-201, 2001.

16. Schneider S, Uchida K, Brabender J, Baldus SE, Yochim J, Danenberg KD, Salonga D, Chen P, Tsao-Wei D, Groshen S, Hoelscher AH, Schneider PM and Danenberg PV: Downregulation of TS, DPD, ERCC1, GST-Pi, EGFR, and HER2 gene expression after neoadjuvant three-modality treatment in patients with esophageal cancer. J Am Coll Surg 200: 336-344, 2005.

17. Salonga D, Danenberg KD, Johnson M, et al: Colorectal tumors responding to 5-fluorouracil have low gene expression levels of dihydropyrimidine dehydrogenase, thymidylate synthase, and thymidine phosphorylase. Clin Cancer Res 6: 1322-1327, 2000.

18. Olaussen KA, Dunant A, Fouret P, et al; IALT Bio Investigators: DNA repair by ERCC1 in non-small-cell lung cancer and cisplatin-based adjuvant chemotherapy. N Engl J Med 355: 983-991, 2006.

19. Tabernero J, Macarulla T, Ramos FJ and Baselga J: Novel targeted therapies in the treatment of gastric and esophageal cancer. Ann Oncol 16: 1740-1748, 2005.

20. Kowanetz M and Ferrara N: Vascular endothelial growth factor signaling pathways: therapeutic perspective. Clin Cancer Res 12: 5018-5022, 2006.

21. Melillo G: Inhibiting hypoxia-inducible factor 1 for cancer therapy. Mol Cancer Res 4: 601-605, 2006.

22. Vallbohmer D and Lenz HJ: Predictive and prognostic molecular markers in outcome of esophageal cancer. Dis Esophagus 19: 425-432, 2006. 